\title{
A Facile Method to Quantify the Carboxyl \\ Group Areal Density in the Active Layer of Polyamide Thin-Film Composite Membranes
}

\author{
Ding Chen ${ }^{\mathrm{a} 1}$, Jay R. Werber ${ }^{\mathrm{b}, \mathrm{c} 1}$, Xuan Zhao ${ }^{\mathrm{a}^{*}}$ and Menachem Elimelech ${ }^{\mathrm{b}, \mathrm{c}^{*}}$ \\ ${ }^{a}$ Collaborative Innovation Center for Advanced Nuclear Energy Technology, INET, Tsinghua \\ University, Beijing 100084, PR China \\ ${ }^{b}$ Department of Chemical and Environmental Engineering, Yale University, New Haven, \\ Connecticut 06520-8286
}

${ }^{c}$ Nanosystems Engineering Research Center for Nanotechnology-Enabled Water Treatment (NEWT), Yale University

zhxinet@tsinghua.edu.cn

menachem.elimelech@yale.edu

"Corresponding author: Xuan Zhao, , Phone: +861062796428

*Corresponding authors: Menachem Elimelech, , Phone: (203) 432-2789

\begin{abstract}
Polyamide thin-film composite (TFC) membranes are the industry standard for membrane-based desalination. Negatively-charged carboxyl groups in the polyamide selective layer play an important role in membrane performance, affecting ion permeation, fouling, and scaling. As such, simple and accurate quantitation of the carboxyl group density is needed. While several methods already exist, each has important drawbacks that limit its application. In this study, we develop a simple bind-and-elute method utilizing silver ion probes, with silver quantitation performed using inductively coupled plasma mass spectrometry. First, the efficacy of the binding, wash, and elution steps is verified, most
\end{abstract}

\footnotetext{
1 These authors contributed equally to this work.
} 
notably using ion exchange resin with known binding capacity. The method is then used to characterize the carboxyl group density and ionization behavior of six commercial polyamide TFC membranes, with total densities ranging from 7.2 to 37 sites $/ \mathrm{nm}^{2}$ and ionization behaviors best described using two acid dissociation constants ( $\mathrm{p} K_{a}$ values). Comparison with data for polyamide layers isolated using dimethylformamide (DMF) shows a 35-65\% decrease in carboxyl density, suggesting that DMF may dissolve uncrosslinked polyamide oligomers. Lastly, the method is used to characterize the effect of two membrane surface modifications, with the results supporting an alternative physical interpretation of the ionization behavior in which a carboxyl group's $\mathrm{p} K_{a}$ is dependent on whether it is located on the surface or buried within the polyamide network. The simplicity, accuracy, and accessibility of the developed method should allow for widespread usage in future studies involving membrane surface charge, as well as studies involving other charged interfaces.

\section{Graphical Abstract}

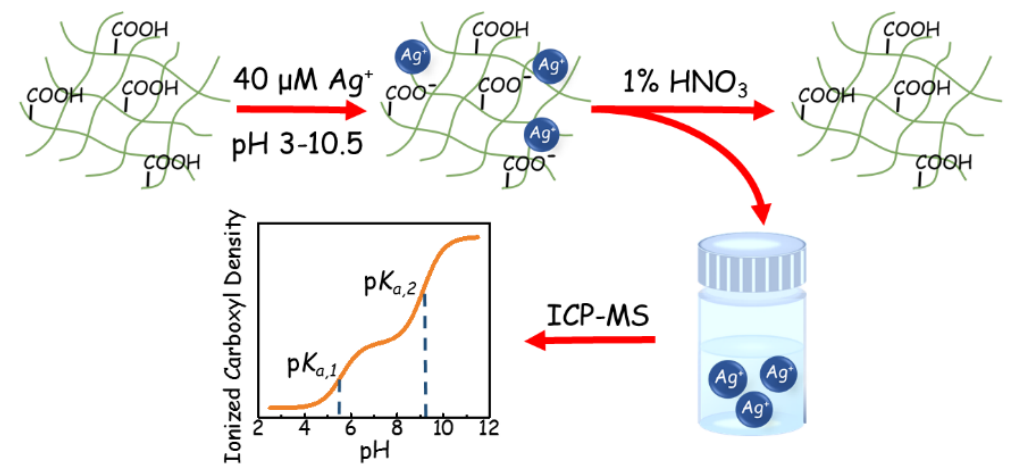

\section{Keywords}

Carboxyl group; polyamide thin-film composite membranes; inductively coupled plasma mass spectrometry; surface charge 


\section{Introduction}

Polyamide thin-film composite (TFC) membranes are state of the art for membrane-based desalination $[1,2]$. TFC membranes are commonly used for pressure-driven processes such as reverse osmosis (RO) and nanofiltration (NF), as well as the osmotically-driven forward osmosis (FO) process. TFC membranes comprise three layers: non-woven fabric backing, polysulfone-based microporous support layer, and a thin, highly-crosslinked polyamide selective layer on the top surface [2]. The polyamide layer is formed through the interfacial polymerization of a diamine, typically $m$-phenylenediamine (MPD), with a tri-acid chloride, typically tri-mesoyl chloride (TMC). The polyamide selective layer is the crucial separation barrier, allowing permeation of water while largely inhibiting permeation of undesired solutes.

TFC membranes are typically negatively charged due the presence of carboxyl groups on the polyamide surface and within the polyamide film. Carboxyl groups are mostly formed from the hydrolysis of acid chloride groups during and following interfacial polymerization. Unreacted amine groups may also be present, but are typically present at lower densities and are mostly unprotonated at $\mathrm{pH}>5$ for MPD-based TFC membranes [3]. The carboxyl density is affected by the conditions of interfacial polymerization. For example, the carboxyl density can be increased by including mono-hydrolyzed TMC as a co-reactant during interfacial polymerization [4]. Post-treatment can also affect the carboxyl density. For example, carboxyl groups are formed from the hydrolysis of amide bonds during hypochlorite treatment, which is commonly used to alter the permeability and alkaline stability of TFC membranes [5, 6].

The charge density in TFC membranes can play an important role in the separation of charged species, especially for NF membranes [7,8]. Due to the looser network structure of NF membranes [9], steric hindrance to solute permeation is reduced, and electrostatic interactions play an increased role in ion rejection [10]. The carboxyl surface charge density can directly influence separation performance in NF. For example, increasing the carboxyl charge density increases the relative rejection of sodium sulfate compared to sodium chloride $[9,11]$. Surface charge is also critical in FO, in which negatively charged groups can facilitate 
cation exchange that dramatically increases levels of bidirectional cation flux [12, 13].

The carboxyl group density in TFC membranes can also influence membrane performance through scaling and fouling. For example, binding of calcium ions to surface carboxyl groups may increase gypsum scaling through enhanced surface nucleation $[14,15]$. Carboxyl-bound calcium ions can also enhance fouling of negatively charged organic foulants, such as alginate and natural organic matter, through the formation of calcium bridges $[16,17]$. Hence, accurate determination of membrane carboxyl group density is of paramount importance.

Several methods have been employed to characterize membrane surface charge, the most common of which is streaming potential analysis [18-20]. However, this method yields the zeta (electrokinetic) potential instead of a quantitative charge density, combines effects from all charged groups on the surface, and often yields negative values for neutral surfaces [21]. Acid-base titration, which is commonly used for colloidal suspensions and dissolved polymers [22], is not sensitive enough for use with TFC membranes.

Table 1 compares other methods to directly or indirectly quantify the carboxyl group density of TFC membranes. Although all methods have been applied with some success, they each have important drawbacks. For example, the method employing the binding and elution of positively charged toluidine blue $\mathrm{O}$ dye is simple and accessible [21]; however, non-specific binding and poor reproducibility hinder this method. The gold-standard method uses silver ions $\left(\mathrm{Ag}^{+}\right)$as an ion probe to bind to negatively charged carboxyl groups, followed by silver quantitation within the film using Rutherford backscattering spectroscopy (RBS) [3, 23-25]. This direct, quantitative measurement has been used to characterize the ionization behavior of several commercial RO and NF membranes. The critical limitation to this method is the inaccessibility of RBS, which is a highly specialized, expensive, and rare instrument.

In this study, we modify the silver-binding procedure used in RBS studies by including a low-pH elution step to yield a simple bind-and-elute method. The eluted silver-and therefore the carboxyl density - is then quantified using inductively coupled plasma mass spectrometry (ICP-MS). We first verify the accuracy and precision of the developed assay, 
most notably through the use of weak cation exchange resin of known binding capacity. To demonstrate the utility of the method, we then analyze the ionized carboxyl density of six commercial RO membranes before and after treatment with dimethylformamide (DMF). We also assess the effect of two surface modification strategies on the ionized carboxyl density, providing insight into the ionization behavior of carboxyl groups. The developed procedure combines the accuracy of RBS-based procedures with the widespread accessibility of ICP-MS instrumentation.

Table 1. Comparison of techniques for the measurement of carboxyl group density of polyamide selective layer (extended from Tiraferri et al. [21])

\begin{tabular}{|c|c|c|c|}
\hline Technique & Advantages & Disadvantages & References \\
\hline $\begin{array}{l}\text { Contact angle } \\
\text { titration }\end{array}$ & $\begin{array}{l}\text { No specific instrumentation } \\
\text { or skills required }\end{array}$ & $\begin{array}{l}\text { Indirect quantification; sensitive } \\
\text { to surface conditions }\end{array}$ & {$[26]$} \\
\hline $\begin{array}{l}\text { Chemical force } \\
\text { titration }\end{array}$ & & $\begin{array}{l}\text { Experimentally difficult; } \\
\text { qualitative; only gives } \\
\text { information on ionization }\end{array}$ & {$[27,28]$} \\
\hline $\begin{array}{l}\text { Quartz crystal } \\
\text { microbalance with } \\
\text { dissipation }\end{array}$ & Repeatable and reproducible & $\begin{array}{l}\text { Difficult sample-handling; } \\
\text { expensive materials and } \\
\text { instrumentation; requires } \\
\text { dissolution of polysulfone, which } \\
\text { may affect measurements }\end{array}$ & {$[29]$} \\
\hline $\begin{array}{l}\text { Rutherford } \\
\text { backscattering } \\
\text { spectroscopy (RBS) }\end{array}$ & $\begin{array}{l}\text { Precise and analytical; } \\
\text { information on ionization } \\
\text { and density of functional } \\
\text { groups }\end{array}$ & $\begin{array}{l}\text { Requires highly specialized and } \\
\text { expensive instrumentation }\end{array}$ & {$[3,23-25]$} \\
\hline $\begin{array}{l}\text { Toluidine blue } \mathrm{O} \\
\text { binding (TBO) }\end{array}$ & Simple; inexpensive & $\begin{array}{l}\text { Poor reproducibility; } \\
\text { non-specific binding }\end{array}$ & {$[21]$} \\
\hline $\begin{array}{l}\text { Uranyl cation } \\
\text { binding }\end{array}$ & $\begin{array}{l}\text { More sensitive than TBO; } \\
\text { provides information for the } \\
\text { entire film }\end{array}$ & $\begin{array}{l}\text { No information about the degree } \\
\text { of ionization; divalent ion, may } \\
\text { not bind 1:1 with functional } \\
\text { groups; requires training and } \\
\text { instrumentation for radioactive } \\
\text { materials }\end{array}$ & {$[21]$} \\
\hline $\begin{array}{l}\text { X-ray photoelectron } \\
\text { spectroscopy (XPS) }\end{array}$ & $\begin{array}{l}\text { O:N ratio allows estimation } \\
\text { of cross-linking ratio; can be } \\
\text { used with ion probes to } \\
\text { quantify carboxyl groups }\end{array}$ & $\begin{array}{l}\text { Only assesses top } \sim 6 \mathrm{~nm} \text { of } \\
\text { membrane; affected by surface } \\
\text { coatings and surface } \\
\text { contamination; high experimental } \\
\text { variability in O:N ratio }\end{array}$ & $\begin{array}{l}{[25,30,} \\
31]\end{array}$ \\
\hline
\end{tabular}




\section{Materials and methods}

\subsection{Chemicals and Membranes}

For analysis in the developed method, six commercial RO membranes from Dow Chemical Company (Midland, MI) and a hand-cast TFC membrane were used. Table 2 lists the commercial membranes used. Fabrication details for the hand-cast membrane are provided in the Supplementary Materials. All chemicals were reagent grade and purchased from Sigma-Aldrich (St. Louis, MO). Nitric acid used in ICP-MS was trace metal grade. All solutions were prepared with deionized (DI) water from a Milli-Q ultrapure water system (Millipore, Billerica, MA).

Table 2. Manufacturer specifications for commercial membranes used in this study

\begin{tabular}{cccccc}
\hline & Application & $\begin{array}{c}\text { Sodium chloride } \\
\text { concentration } \\
(\mathrm{mg} / \mathrm{L})\end{array}$ & $\begin{array}{c}\text { Applied } \\
\text { pressure } \\
(\mathrm{bar})\end{array}$ & $\begin{array}{c}\text { Permeate } \\
\text { Flux } \\
\left(\mathrm{Lm}^{-2} \mathrm{~h}^{-1}\right)\end{array}$ & $\begin{array}{c}\text { Stabilized } \\
\text { Salt Rejection } \\
(\%)\end{array}$ \\
\hline SW30XLE & Seawater & 32000 & 55 & 38.2 & 99.8 \\
BW30 & Brackish water & 2000 & 15.5 & 44.6 & 99.5 \\
BW30FR & Brackish water & 2000 & 15.5 & 44.6 & 99.5 \\
BW30XFR & Brackish water & 2000 & 15.5 & 48.8 & 99.65 \\
LE & $\begin{array}{c}\text { Low pressure } \\
\text { brackish water }\end{array}$ & 2000 & 10.3 & 48.8 & 99.3 \\
Low pressure & 2000 & 8.6 & 54.0 & 99.0 \\
\hline
\end{tabular}

\subsection{Membrane preparation}

Membrane samples were first wetted in $25 \%$ isopropanol for 30 minutes, after which the polyester fabric backings were physically removed. The remaining polyamide/polysulfone membranes were rinsed twice in DI water to remove residual isopropanol, and stored in DI water before use. For some samples, polyamide films were isolated by dissolution of the polysulfone support in dimethylformamide (DMF) [32]. The isolated polyamide layer was gently transferred using tweezers and immersed two more times in fresh DMF to ensure 
complete removal of polysulfone. The polyamide film was then rinsed at least twice in DI water to remove residual DMF, and stored in DI water before use. Unless otherwise indicated, membrane samples were $2.0 \mathrm{~cm}^{2}$ in area.

\subsection{Binding and elution of silver ion probes}

Silver ion binding methods were adapted from the procedure reported by Coronell et al. [3]. Membranes and isolated polyamide films were immersed twice, each for $10 \mathrm{~min}$, in 10 $\mathrm{mL}$ of $40 \mu \mathrm{M}$ silver nitrate at the desired $\mathrm{pH}$. For analysis of ionization behavior, samples were typically assessed at $\mathrm{pH} 3.5,5,7,9,10$, and 10.5. Silver is fully soluble at the binding concentration of $40 \mu \mathrm{M}$ over the given $\mathrm{pH}$ range. After the binding step, membranes and isolated polyamide films were immersed four times, each for $7 \mathrm{~min}$, in $10 \mathrm{~mL}$ of $1 \mu \mathrm{M}$ silver nitrate at the same $\mathrm{pH}$ used during binding to rinse off unbound silver. Membranes were touched lightly against filter paper after each step to minimize solution carryover. Sodium hydroxide and nitric acid were used to adjust the $\mathrm{pH}$. Following the wash steps, membranes and isolated polyamide films were immersed in $5 \mathrm{~mL}$ of $1 \%$ nitric acid for $30 \mathrm{~min}$ to protonate the carboxyl groups and elute the bound silver ions. After physical removal of the silver-free membranes and polyamide films, solutions were ready for ICP-MS analysis.

\subsection{Silver ion quantitation using ICP-MS}

ICP-MS (ELAN DRC-e, Perkin Elmer, Waltham, MA) was used to quantify the silver ion concentration. Nitric acid solutions (1\%) with silver concentrations ranging from 0-200 $\mu \mathrm{g} / \mathrm{L}$ were used to calibrate the system. Indium nitrate $(40 \mu \mathrm{g} / \mathrm{L})$ was used as an internal standard for ICP-MS. To convert the silver concentration to areal carboxyl group density, it was assumed that each eluted silver ion corresponded to one ionized carboxyl group (i.e., 1:1 binding), as in RBS-based methods [3]. It was also assumed that silver ions can access all carboxyl groups within the polyamide film [3]. Therefore, the concentration of ionized carboxyl was determined using:

$$
\left[R-C O O^{-}\right]=\frac{C_{A g^{+}} V_{A g^{+}} N_{A}}{A}
$$

where $\left[\mathrm{R}-\mathrm{COO}^{-}\right]$is the ionized carboxyl group areal density (number of groups per unit area), $A$ is the projected surface area of the polyamide film, $C_{A g+}$ is the silver molar concentration 
measured in ICP-MS, $V_{A g+}$ is the elution volume, and $N_{A}$ is Avogadro's number. The projected surface area is defined as the single-sided surface area of the membrane sample, neglecting surface roughness.

\subsection{Verification of binding and wash steps using ion exchange resin and acid-base titration}

Carboxyl-based weak acid ion exchange (IEX) resin (Dowex MAC-3, Dow Chemical Company, Midland, MI) was used to verify (i) 1:1 binding of silver with ionized carboxyl groups and (ii) negligible removal of bound silver during wash steps when sodium ions are present. The ion exchange capacity of the IEX resin was determined using Boehm titration procedures [33]. IEX resin was first immersed in $1 \%$ nitric acid to fully acidify the resin before drying in an oven at $60^{\circ} \mathrm{C}$. Then, $400 \mathrm{mg}$ of acidified resin was mixed with $20 \mathrm{~mL}$ sodium bicarbonate with a concentration of $0.2 \mathrm{M}$. After equilibrating for $3 \mathrm{~h}, 5 \mathrm{~mL}$ of the supernatant was transferred into $20 \mathrm{~mL}$ DI water and titrated to $\mathrm{pH} 3.1$ using $0.12 \mathrm{M}$ hydrochloric acid. As a control, $5 \mathrm{~mL}$ of $0.2 \mathrm{M}$ sodium bicarbonate was also titrated. The ion-exchange capacity was calculated using:

$$
C=\frac{4\left(V_{\text {Control }}-V_{\text {Resin }}\right) C_{H C l}}{m}
$$

where $C$ is the ion exchange capacity, $V_{\text {Resin }}$ is the volume of acid used for resin titration, $V_{\text {Control }}$ is the acid volume used for control titration, $C_{H C l}$ is the molar concentration of acid, and $m$ is the mass of dry resin used for titration.

For the silver binding experiment, $2.0 \mathrm{mg}$ resin was incubated three times, each for 1 day, in $50 \mathrm{~mL}$ of $5 \mathrm{mM}$ silver nitrate at $\mathrm{pH} 8.5$, with aliquots of $1 \mathrm{M}$ sodium hydroxide added after resin addition to maintain the desired $\mathrm{pH}$. Elevated silver nitrate volumes and concentrations were used due to the high ion exchange capacity of the IEX resin. Wash and elution steps were as described in Section 2.2, with the wash step conducted at $\mathrm{pH}$ 8.5. The eluted silver solution was diluted 1:10,000 with $1 \%$ nitric acid to get within the working range of the ICP-MS.

\subsection{Verification of complete silver ion elution using microwave digestion}

Microwave digestion using a MARS 5 digestor (CEM Corporation, Matthews, NC) was used to verify the silver ion elution method. Isolated polyamide layers were treated with silver at $\mathrm{pH} 10.5$ using the method described in Section 2.3, without the low-pH elution step. 
Silver-treated membrane samples were loaded in 100-mL microwave tubes (XP-1500 plus vessels) with $2 \mathrm{~mL}$ of concentrated nitric acid and $4 \mathrm{~mL}$ of DI water, resulting in a final nitric acid concentration of $20-23 \%$. The membrane samples were then digested following the procedure described in Table 3 [34]. No visible residue remained in the tube after digestion. After the system reached ambient temperature, samples were removed and diluted to $50 \mathrm{~mL}$ with DI water. The silver concentration in the samples was then measured by ICP-MS. To ensure that no silver was lost during the microwave digestion process, a spike recovery test was performed using $0.5 \mathrm{~mL}$ of $10 \mathrm{mg} / \mathrm{L}$ silver nitrate in $1 \%$ nitric acid.

Table 3. Microwave digestion protocol [34]

\begin{tabular}{cccccc}
\hline Stage & Power & $\begin{array}{r}\text { Ramp time } \\
(\mathrm{min})\end{array}$ & $\begin{array}{c}\text { Pressure } \\
(\mathrm{bar})\end{array}$ & $\begin{array}{c}\text { Temperature } \\
\left({ }^{\circ} \mathrm{C}\right)\end{array}$ & $\begin{array}{c}\text { Hold time } \\
(\mathrm{min})\end{array}$ \\
\hline 1 & 600 & 5 & 5.0 & 175 & 3.0 \\
2 & 1200 & 10 & 10.0 & 210 & 10.0 \\
\hline
\end{tabular}

\subsection{Surface modification procedures}

SW30XLE membranes without fabric backing were used for surface modification, with modification procedures shown schematically in Fig. 1. The reaction of ethylamine with residual carboxyl groups on the membrane surface was mediated by the activating reagents 1-ethyl-3-(3-dimethylaminopropyl)carbodiimide hydrochloride (EDC) and $N$-hydroxysuccinimide (NHS), following previously published procedures [35]. In short, membranes were placed in $20 \mathrm{~mL}$ of EDC-NHS activation solution (EDC and NHS were 4 $\mathrm{mM}$ and $10 \mathrm{mM}$, respectively) prepared with MES buffer solution (10 mM MES, 0.5 M NaCl, $\mathrm{pH} 5$ ) and shaken for $1 \mathrm{~h}$ at room temperature. After being rinsed in DI water, membranes were immersed in $20 \mathrm{~mL}$ of $10 \mathrm{mM}$ ethylamine, prepared with HEPES buffer solution (10 mM HEPES, $0.15 \mathrm{M} \mathrm{NaCl}, \mathrm{pH} 7.5$ ), and shaken for another $0.5 \mathrm{~h}$. Finally, membranes were removed, thoroughly rinsed with DI water, and stored in DI water.

Grafting of polyacrylic acid followed previously published procedures [36]. In short, a 1 $\mathrm{M}$ acrylic acid monomer solution was prepared and mixed with $10 \mathrm{mM}$ potassium persulfate 
and $10 \mathrm{mM}$ sodium metabisulphite as initiators. Grafting was carried out by sealing the membrane such that only the active layer was exposed, then covering the membrane with 20 $\mathrm{mL}$ of reaction medium for $1 \mathrm{~h}$ at room temperature. The membrane was then carefully washed with DI water to remove unreacted monomer and free polyacrylic acid. The modified membrane was then stored in DI water until further use.

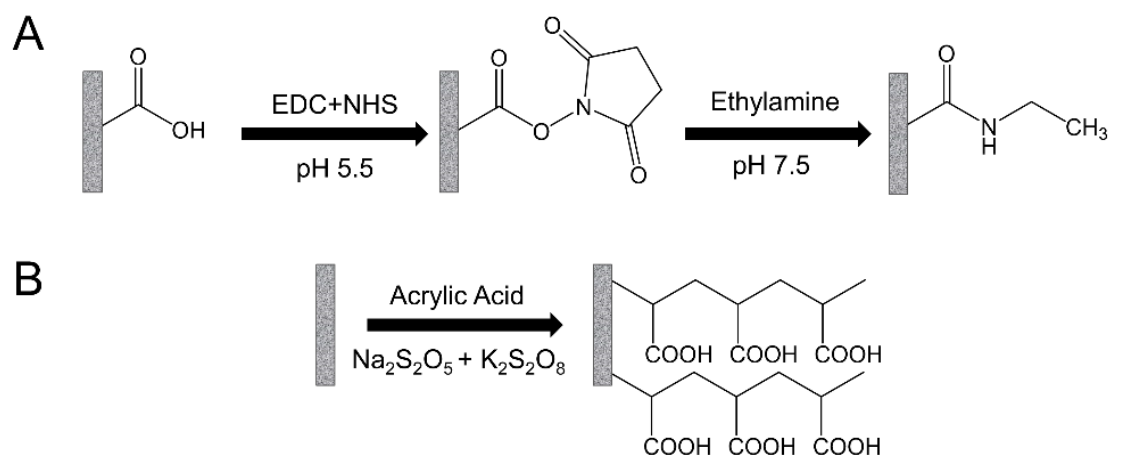

Fig. 1. Surface modification procedures that were assessed using the developed assay. (A) Condensation reaction of ethylamine with residual carboxyl groups, mediated by 1-ethyl-3-(3-dimethylaminopropyl)carbodiimide (EDC) and $N$-hydroxysuccinimide (NHS). (B) Redox-initiated polymerization of polyacrylic acid from the active layer surface. The starting membrane for each reaction was SW30XLE without the fabric backing.

\section{Results and discussion}

\subsection{Verification of bind-and-elute procedure}

\subsubsection{Assay linearity}

Commercial RO membrane samples (SW30XLE) were used to test the assay procedure. As described in Section 2.2, the polyester fabric backing was physically removed prior to the silver-binding procedure. Due to the large mass of polyester relative to polyamide, minor rates of ester-bond hydrolysis could lead to significant levels of carboxyl groups, as was indeed found for the fabric backing of BW30 membranes (Fig. S1). Silver binding to polysulfone was also considered, but was found to be negligible. The effect of polysulfone is discussed further in Section 3.3.

To test assay linearity, SW30XLE samples were treated with silver nitrate at $\mathrm{pH} 10.5$, 
following the method in Section 2.3, and eluted in $1 \%$ nitric acid. The eluted silver increased linearly with increasing membrane area (Fig. 2), yielding a consistent ionized carboxyl areal density of 9-10 sites $/ \mathrm{nm}^{2}$. The steps for binding silver and washing excess silver from the membrane were adapted from previous RBS studies [3], except that the silver binding concentration was kept constant in this study for simplicity. Increased binding time, increased binding concentration, and increased elution time were not found to meaningfully impact results (Table S1).

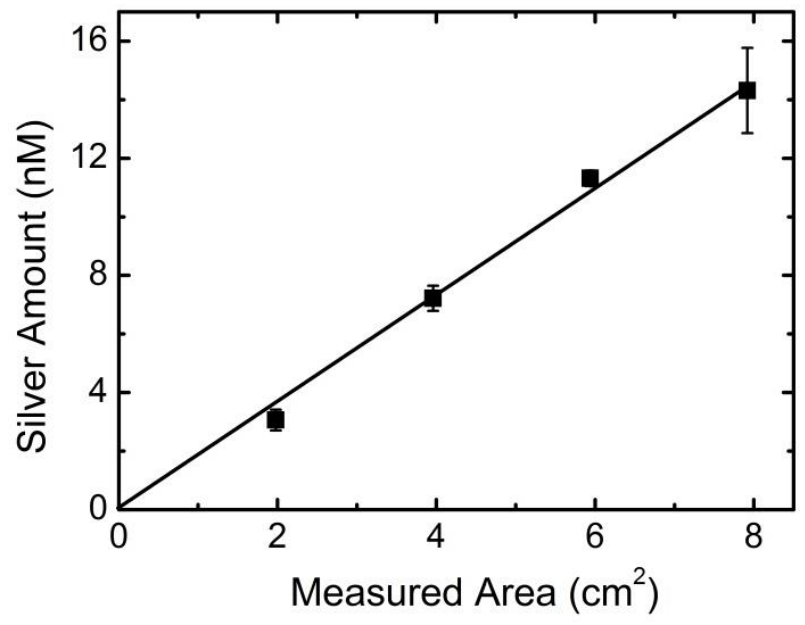

Fig. 2. Measured silver amount versus area of the membrane sample. All samples were SW30XLE with the polyester fabric removed, and were assessed with binding and wash steps conducted at $\mathrm{pH} 10.5$.

\subsubsection{One-to-one silver binding and minimal sodium binding}

The carboxyl density quantitation method used in this study and developed in RBS-based studies is based on the assumption that monovalent silver ions bind 1:1 with ionized carboxyl groups [3]. In addition, it was assumed that binding of sodium is negligible since ion exchange processes show a strong affinity for $\mathrm{Ag}^{+}$over $\mathrm{Na}^{+}$. In the binding and wash solutions, sodium can be present at greater levels than silver, especially at high $\mathrm{pH}$, due to the addition of sodium hydroxide to achieve the desired $\mathrm{pH}$.

To verify that these two assumptions hold true, the silver binding method was compared with acid-base titration for a commercial carboxylate-based cation exchange resin (IEX, Dowex MAC-3), as shown in Fig. 3A. Titration yielded an ion exchange capacity of 5.9 \pm 0.1 meq/g dry resin, which is comparable to the manufacturer specification of $3.8 \mathrm{meq} / \mathrm{mL}$ wet 
resin. Silver binding gave a similar ion exchange capacity of $5.7 \pm 0.9 \mathrm{meq} / \mathrm{g}$ dry resin. The equivalent capacities measured by the two methods indicate that 1:1 binding does occur for IEX resin and that sodium binding is negligible. The equivalent capacities also indicate the effectiveness of the silver-binding protocol.

In order to translate the results for IEX resin to polyamide films, the accessibility of silver ions to carboxyl groups located in small pores must be considered. Addressing this issue, Coronell et al. [3] noted that according to ion-exchange theory the ionic radius becomes the relevant dimension for rigid porous structures (e.g., highly crosslinked polymers) if the hydrated radius is too large to allow entry into the pores. Since the ionic radius of $\mathrm{Ag}^{+}$ $(0.08-0.14 \mathrm{~nm})$ is less than the size of network pores within polyamide films $(0.14-0.23 \mathrm{~nm}$, as measured in the dry state by positron annihilation lifetime spectroscopy [37]), it was assumed that complete accessibility does occur. In addition, the pore size increases slightly upon film hydration $[38,39]$, further strengthening this assumption.

To experimentally verify the assumption of complete accessibility, the carboxyl density measured using the silver-binding method would need to be compared with densities obtained using a quantitative, probe-free analytical method, which has yet to be developed. Development of a probe-free method is out of the scope of this study. To gain experimental insight into silver accessibility, filtration experiments using SW30XLE membranes were conducted at $15.5 \mathrm{bar}, 25^{\circ} \mathrm{C}$, and $25 \mathrm{~cm} / \mathrm{s}$ crossflow velocity, obtaining an observed rejection of just $29 \pm 6 \%$ for a $1.8 \mu \mathrm{M}$ silver nitrate (194 $\mu \mathrm{g} / \mathrm{L}$ silver) feed solution (Table S2). The relatively high permeability of silver ions indicates that silver readily penetrates the polyamide film. Based on this high permeability and the rationale discussed above, we assume that silver ions are completely accessible to ionized carboxyl groups within TFC membranes. As such, the measured silver binding density for silver-treated membranes is assumed to correspond 1:1 with the ionized carboxyl density.

\subsubsection{Acidic elution of silver ion probes}

Another concern in this method is whether silver ions completely elute in $1 \%$ nitric acid (i.e., whether ionized carboxyl groups electrostatically bound to silver effectively re-protonate at low $\mathrm{pH}$ ). To ensure that complete elution occurs, the acidic elution step was 
compared with microwave digestion. Microwave digestion is a common technique used to dissolve metal species present in solid samples by chemically degrading the solid sample in a highly acidic solution [40]. Polyamide films isolated from SW30XLE by dissolution in DMF were bound with silver at $\mathrm{pH} 10.5$, washed, then either immersed in $1 \%$ nitric acid or fully degraded by microwave digestion. As shown in Fig. 3B, the measured silver was nearly identical between the two methods. A spike recovery test using $5 \mathrm{mg} / \mathrm{L}$ silver nitrate was also performed with $97.3 \pm 9.5 \%$ recovery, indicating that no silver is lost during microwave digestion. The results suggest that silver ions bound to the polyamide film are effectively eluted with $1 \%$ nitric acid.
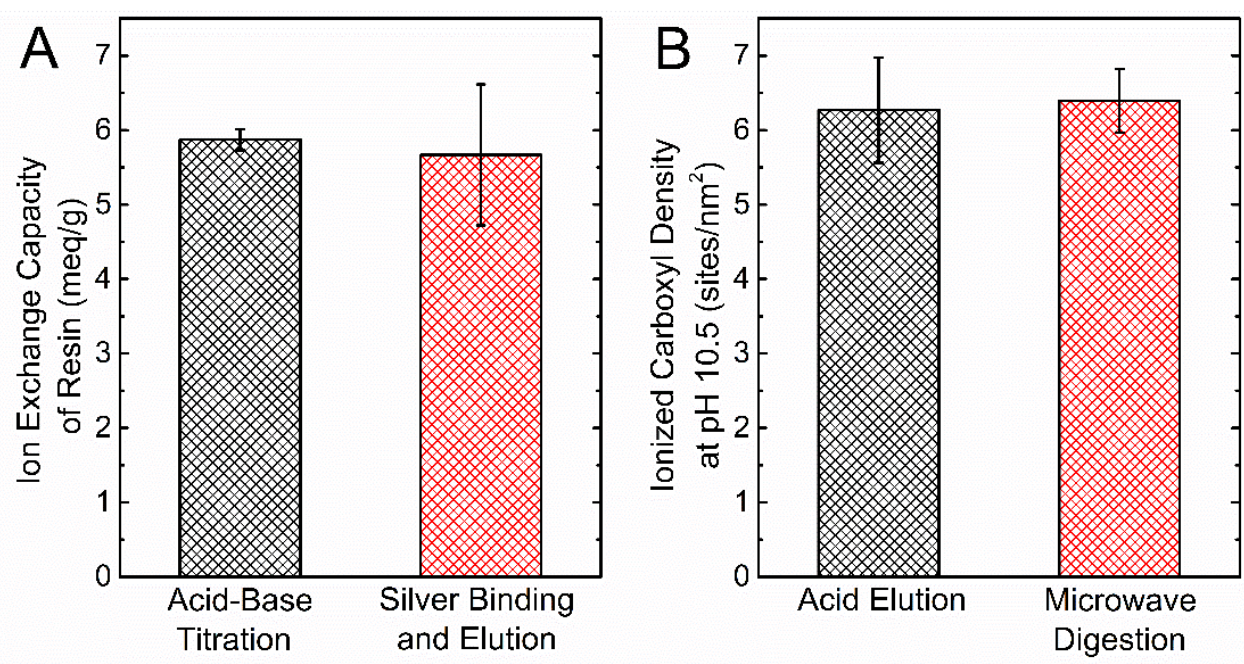

Fig. 3. (A) Verification of binding and wash steps using carboxyl-based weak acid ion exchange resin (Dowex MAC-3, Dow Chemical). Acid-base titrations followed standard procedures to measure ion exchange capacity, as described in Section 2.5. Silver binding was conducted at pH 8.5. (B) Comparison of the measured ionized carboxyl density of isolated polyamide films using two different elution techniques. Acid elution used $1 \%$ nitric acid to elute silver while leaving the polyamide film intact, following the method described in Section 2.2. Microwave digestion completely degraded the polyamide film, following the method described in Section 2.4. For both elution conditions, binding and wash steps were conducted at $\mathrm{pH} 10.5$ (pH adjusted with sodium hydroxide).

\subsection{Carboxyl density and ionization behavior of commercial membranes}

Following verification of the procedure, the carboxyl group areal density and ionization 
behavior were assessed for six commercial TFC membranes used for seawater RO, brackish water RO, and low pressure brackish water RO (Fig. 4). These membranes may include a hydrophilic coating layer, most likely the neutral polyvinyl alcohol [31]. Coating layers on commercial TFC membranes have not been found to bind cation probes [31], including silver [3], and as such are unlikely to affect measurements of carboxyl group densities. Carboxyl group ionization was fitted using [3]:

$$
[R-C O O-]=C_{T, R-\mathrm{COOH}} \sum_{i=1}^{n} w_{i} \frac{K_{a, i}}{\left[H^{+}\right]+K_{a, i}}
$$

where $C_{T, R-\mathrm{COOH}}$ is the total carboxyl density, $\left[\mathrm{H}^{+}\right]$is the hydrogen molar concentration, $K_{a}$ is the acid-base equilibrium constant of the carboxyl group, and $w$ is the fraction of carboxyl groups with different $K_{a}$. As done previously [3], two $K_{a}$ values (i.e., $n=2$ in Eq. 3) were used to fit the ionization behavior of each membrane.

Parameters used to fit the data for each membrane are listed in Table 4. The total carboxyl group densities ranged from 7.2 to 37 sites $/ \mathrm{nm}^{2}$, similar to values reported by other methods [3, 21, 23-25, 29]. Since each carboxyl group suggests a missed amide bond, the total carboxyl density gives an indication of the degree of polymer cross-linking, with lower density indicating more cross-linking. For the six membranes tested here, the total carboxyl density somewhat corresponded to the expected "looseness" of the polyamide films, as the seawater and brackish water RO membranes had the lowest values while the low pressure brackish water RO membranes had the highest. However, this may be a coincidence as a previous RBS-based study assessing commercial RO and NF membranes found no link between carboxyl density and membrane application type [23].

In RBS-based studies, the atomic composition of the polyamide film is simultaneously measured with the carboxyl density [3]. Using this composition and the carboxyl density, the degree of polymer cross-linking can be quantitatively calculated. As only carboxyl density is measured in the method here, the degree of cross-linking can only be crudely estimated. A quartz crystal microbalance study assessing the polyamide films of commercial RO membranes found an average polyamide mass of $15400 \mathrm{ng} / \mathrm{cm}^{2}$ [41]. For a completely cross-linked polyamide film, the ratio of TMC monomers to MPD monomers used to form 
the film is 2:3. Using this TMC:MPD ratio, the average mass of $15400 \mathrm{ng} / \mathrm{cm}^{2}$ corresponds to $\sim 286$ TMC units per $\mathrm{nm}^{2}$ of projected surface area, or $\sim 858$ potential amide bonds per $\mathrm{nm}^{2}$. Thus, comparing the total carboxyl densities measured for the six commercial membranes to the number density of potential amide bonds, the degree of cross-linking can be estimated as varying from 95.7-99.2\%. Alternatively, assuming all TMC groups within the film have either 0 or 1 carboxyl group, the fraction of TMC groups that are fully cross-linked can be estimated as varying from $87.0 \%-97.5 \%$.

The fitted $\mathrm{p} K_{a}$ values of the six commercial membranes were very similar (Table 4); $\mathrm{p} K_{a, 1}$ varied from 5.2-5.9, while $\mathrm{p} K_{a, 2}$ varied from 9.1-9.7. As found in RBS studies for most membranes tested, the majority of carboxyl functional groups were not ionized at neutral $\mathrm{pH}$ but were ionized above $\mathrm{pH}$ 9. This phenomenon matches well with studies on bidirectional cation exchange in FO, which found a dramatic increase in potassium/sodium exchange at higher $\mathrm{pH}(8-10)$ that was attributed to the high negative surface charge at these $\mathrm{pH}$ levels [12].

The first and second $\mathrm{p} K_{a}$ values are slightly and substantially greater, respectively, than the $\mathrm{p} K_{a}$ of 4.2 for benzoic acid, the approximate molecular analog for a carboxyl group in a polyamide film. A recent study speculated that the $\mathrm{p} K_{a}$ shifts to higher values because of the low dielectric constant of the polyamide polymer compared to bulk water [3]. The authors further hypothesized that the degree of the $\mathrm{p} K_{a}$ shift depends on the size of the pore in which the carboxyl group is situated. According to their hypothesis, $\mathrm{p} K_{a, 1}$ corresponded to carboxyl groups within larger aggregate pores $(0.35-0.41 \mathrm{~nm}$ radius) where the greater presence of water increases the dielectric constant, while $\mathrm{p} K_{a, 2}$ corresponded to carboxyl groups within smaller network pores $(0.14-0.23 \mathrm{~nm}$ radius). If this interpretation were correct, one would expect looser membranes to have greater fractions of carboxyl groups in $\mathrm{p} K_{a, 1}$ and/or $\mathrm{p} K_{a}$ values shifted to lower values. However, there are no clear trends in this study or previous studies [23] between the expected permselectivity of the membranes and the $\mathrm{p} K_{a}$ values or the fraction of carboxyl groups in $\mathrm{p} K_{a, 1}$.

Another possible interpretation is that $\mathrm{p} K_{a, 1}$ corresponds to carboxyl groups on the membrane surface (i.e., groups interacting with bulk water), while $\mathrm{p} K_{a, 2}$ corresponds to 
groups buried within the polyamide polymer network, regardless of the local pore structure. This interpretation is consistent with the lack of trends observed between expected permselectivity and ionization behavior for different NF and RO membranes [23]. For the six commercial RO membranes tested here, the carboxyl density at $\mathrm{pH} 7$ varied from 1.7 to 6.1 sites $/ \mathrm{nm}^{2}$. It is physically realistic that these densities could occur on the polyamide surface, especially when considering that the actual polyamide frontal surface has been shown by microscopy to be 4-7 times higher than the projected surface area due to the roughness of the polyamide film [42].

Different $\mathrm{p} K_{a}$ values for surface-bound and buried carboxyl groups could arise from two possible mechanisms. First, the increased $\mathrm{p} K_{a}$ of buried carboxyl groups could stem from a decreased dielectric constant within the film, similar to the hypothesis discussed earlier [3]. Surface-bound carboxyl groups would experience a dielectric constant close to that of the bulk solution, and thus have a lower $\mathrm{p} K_{a}$. Alternatively, the elevated $\mathrm{p} K_{a}$ values of buried groups could be due to the local $\mathrm{pH}$ within the film being lower than the bulk solution $\mathrm{pH}$. Such behavior is observed for ion exchange resins due to the high concentration of bound acidic functional groups, with the disparity in $\mathrm{pH}$ being most pronounced at low solution counter-ion concentration [43]. For example, due to decreased intra-resin $\mathrm{pH}$, the observed $\mathrm{p} K_{a}$ based on solution $\mathrm{pH}$ for a methacrylic acid-based resin is 9.4 when titrated by sodium hydroxide addition to deionized water, substantially greater than the intrinsic $\mathrm{p} K_{a}$ of 4.9 [43]. Considering the low silver concentrations used in the silver-binding method and the relatively high carboxyl group concentrations within the polyamide film $(0.2-0.7 \mathrm{M}$, relative to total film volume $[3,23,25])$, local $\mathrm{pH}$ effects may be responsible for the observed $\mathrm{p} K_{a}$ values in this study and RBS-based studies. A slight decrease in local $\mathrm{pH}$ at the membrane surface and a substantial decrease in local $\mathrm{pH}$ within the polyamide film would explain $\mathrm{p} K_{a, 1}$ and $\mathrm{p} K_{a, 2}$, respectively. Further evidence in support of the surface/buried interpretation is discussed in Section 3.4. 


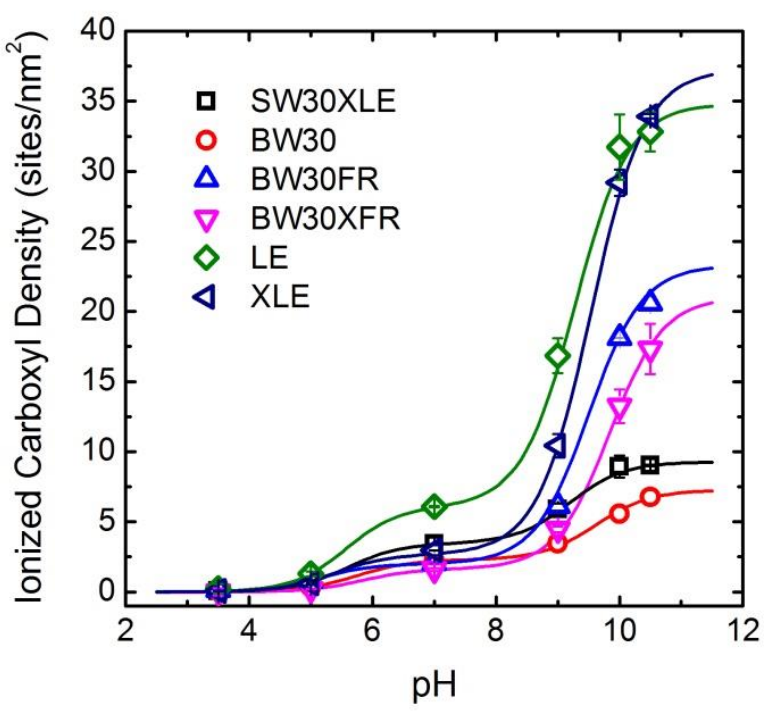

Fig. 4. Ionized carboxyl group density of six commercial reverse-osmosis membranes as a function of $\mathrm{pH}$. The curves were fitted to the data using Eq. 3 and the fitting parameters shown in Table 4. Data was measured using membranes after removal of the polyester fabric backing.

Table 4. Fitting parameters used to fit ionized carboxyl densities for commercial reverse-osmosis membranes. Fitting analysis was performed using Eq. 3 in Origin (OriginLab, Northampton, MA). Error bars reflect uncertainties in the fitting parameters.

\begin{tabular}{ccccc}
\hline & $\mathbf{p} \boldsymbol{K}_{a, 1}$ & $\mathbf{p} \boldsymbol{K}_{a, 2}$ & $\begin{array}{c}\text { Fraction of carboxyl } \\
\text { groups in peak 1 }\end{array}$ & $\begin{array}{c}\text { Total carboxylic group } \\
\text { areal density (sites/nm }\end{array}$ \\
\hline SW30XLE & $5.6 \pm 0.1$ & $9.1 \pm 0.1$ & $0.38 \pm 0.02$ & $9.26 \pm 0.04$ \\
BW30 & $5.6 \pm 0.2$ & $9.6 \pm 0.1$ & $0.32 \pm 0.02$ & $7.2 \pm 0.3$ \\
BW30FR & $5.2 \pm 0.4$ & $9.5 \pm 0.1$ & $0.09 \pm 0.01$ & $23 \pm 1$ \\
BW30XFR & $5.86 \pm 0.01$ & $9.74 \pm 0.01$ & $0.08 \pm 0.01$ & $19.9 \pm 0.2$ \\
LE & $5.57 \pm 0.04$ & $9.2 \pm 0.1$ & $0.18 \pm 0.01$ & $34.5 \pm 0.8$ \\
XLE & $5.4 \pm 0.3$ & $9.5 \pm 0.1$ & $0.07 \pm 0.02$ & $37 \pm 1$ \\
\hline
\end{tabular}

\subsection{Influence of polysulfone and DMF treatment on silver-binding density}

In RBS studies, silver was found to bind at very low rates $(0.006 \%$ atom/atom $)$ to polysulfone, even though polysulfone is typically a neutral polymer [3]. To test whether this low rate of binding could affect the measured carboxyl density, the silver binding density was measured for various components of a hand-cast TFC membrane. The TFC membrane was formed by interfacial polymerization of MPD and TMC on a commercial polysulfone 
ultrafiltration membrane (Sepro PS20, Oceanside, CA) with a polyethylene fabric backing, with fabrication details in the Supplementary Materials. Silver binding densities were measured at $\mathrm{pH} 10.5$ for (i) isolated polyamide films, (ii) isolated fabric backings, (iii) fabric with the polysulfone support layer, and (iv) the full TFC membrane (Fig. 5). Due to mechanical fragility of the polysulfone layer, the fabric could not be physically removed and was thus isolated by dissolution of polysulfone using DMF. Comparison of the fabric-only and polysulfone/fabric samples indicates that either the amount of silver that binds to polysulfone is negligible or the silver ions bound to polysulfone do not elute in $1 \%$ nitric acid. In other words, the presence of polysulfone does not affect the quantitation of carboxyl density in the polyamide layer.

The data additionally shows that immersion into DMF significantly decreases the carboxyl density of the polyamide layer. The carboxyl density for polyamide layers isolated by DMF dissolution was $13.1 \pm 1.8$ sites $/ \mathrm{nm}^{2}, 43.3 \%$ less than the density $(23.1 \pm 0.9$ sites $/ \mathrm{nm}^{2}$ ) calculated for the polyamide layer by subtracting the value for the support layer from that of the full TFC membrane. To ascertain if this was a universal phenomenon, the carboxyl density and ionization behavior of the commercial membranes tested in Section 3.2 were measured after immersion in DMF (Fig. 6). Similar relative decreases $(34.7-64.5 \%)$ in total carboxyl density were observed, with the absolute difference varying widely from 2.8 20.2 sites $/ \mathrm{nm}^{2}$. No trends were observed for ionization; the decrease in carboxyl density was associated with $\mathrm{p} K_{a, 1}$ for some membranes and with $\mathrm{p} K_{a, 2}$ for others.

The most likely explanation for the decreased carboxyl density for all membranes tested is the dissolution of uncrosslinked polyamide oligomers that were physically (not covalently) associated with the crosslinked polyamide film. Recent simulations demonstrated that oligomers are formed in the initial stages of interfacial polymerization, after which the oligomers merge to form an integral polyamide film [45]. Some of these simulated oligomers were found to have an excess of acyl chloride groups, which would result in carboxyl groups following hydrolysis. Dissolution of some of these carboxyl-rich oligomers by DMF would explain our observed decrease in carboxyl group density. Our results corroborate a previous study that found that molecules between 1700 and $11700 \mathrm{Da}$ (measured by gel permeation 
chromatography) come off the TFC surface after immersion in DMF [46].

The finding of oligomer dissolution may be important for other research and membrane-fabrication studies. DMF is commonly used in research studies to isolate polyamide layers for fundamental characterization [32,44]. It is also used in the field of organic solvent nanofiltration to increase the solvent permeability of TFC membranes [30, 46]. As minimal changes in mass, morphology, and chemical properties were observed in previous studies using conventional characterization techniques, the permeability increase has been hypothesized to be due to a solvent effect of DMF on polyamide, with DMF allowing for the polyamide network to rearrange into a more permeable structure [30]. The results here indicate that the removal of oligomers may also play a role.

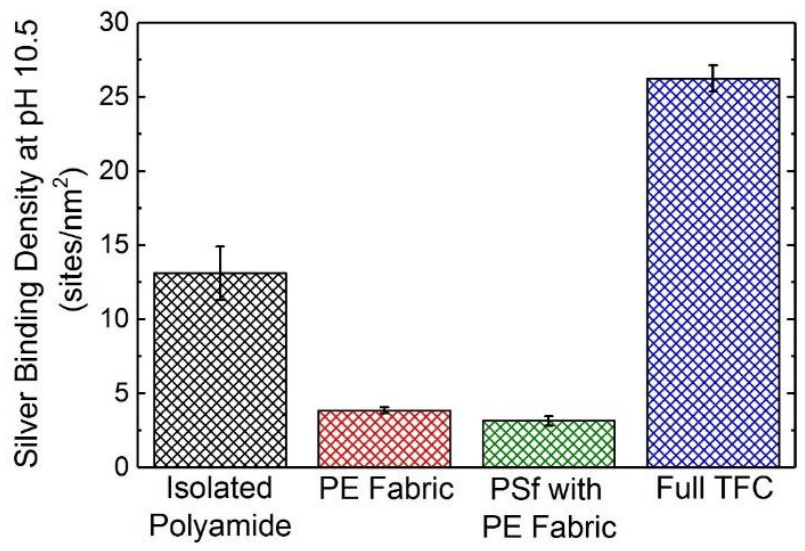

Fig. 5. Effect of dimethylformamide (DMF) dissolution on silver binding density. Silver binding density was measured at $\mathrm{pH} 10.5$ for hand-cast thin-film composite (TFC) membranes formed via the interfacial polymerization of $m$-phenylenediamine and tri-mesoyl chloride on a commercial polysulfone (PSf) ultrafiltration membrane (PS20, Sepro Membranes, Inc., Oceanside, CA) with polyethylene (PE) fabric backing. For comparison, binding densities were also measured for the isolated PE fabric, the polysulfone support layer with the PE fabric, and for isolated polyamide films following DMF dissolution. The fabric could not be physically removed due to fragility of the polysulfone layer, and was thus isolated using DMF dissolution of polysulfone. Details on interfacial polymerization are given in Supplementary Materials. 

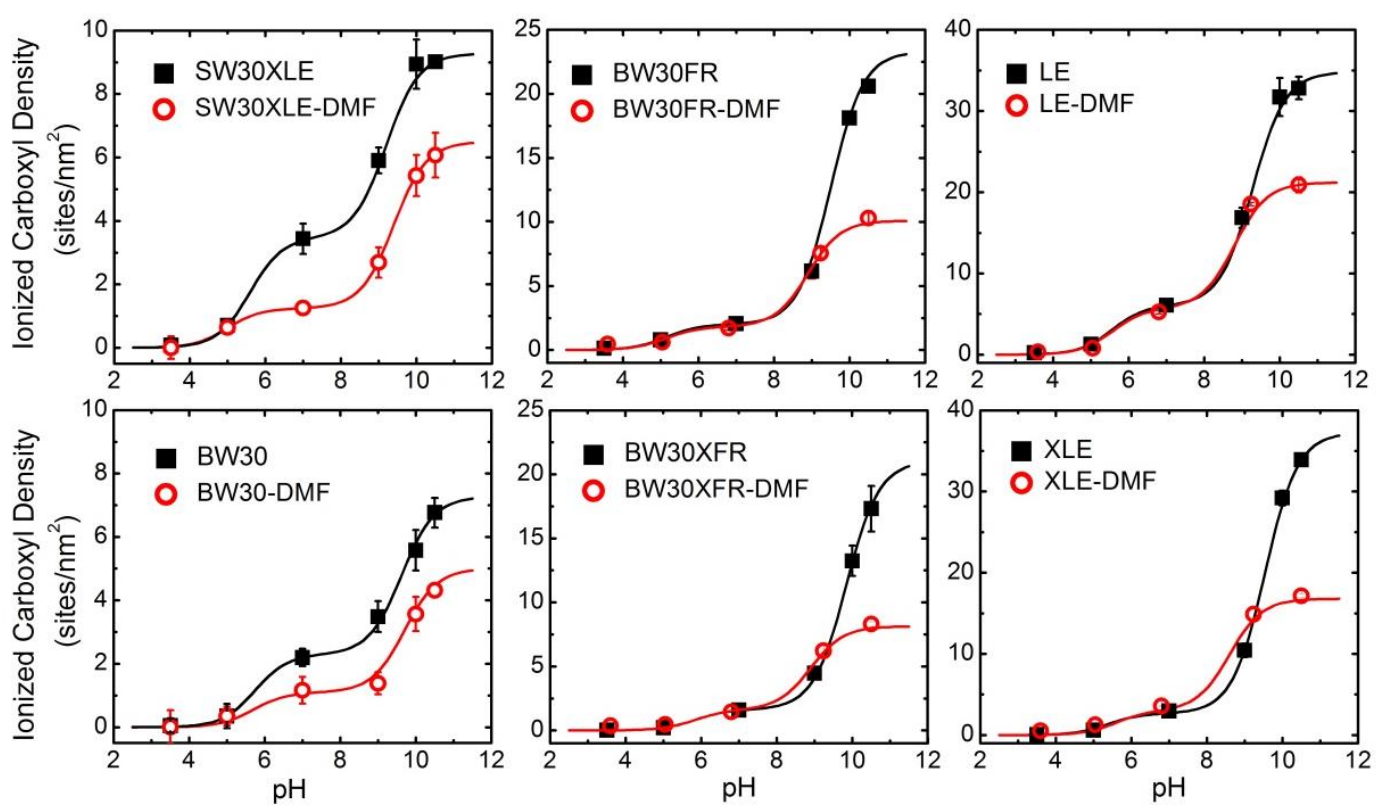

Fig. 6. Ionized carboxyl density as a function of $\mathrm{pH}$ for commercial reverse-osmosis membranes with and without dimethylformamide (DMF) treatment. The polyester fabric backing was removed for all samples. The membranes after DMF treatment are pure polyamide films. Curves were fit using Eq. 3 assuming two $\mathrm{p} K_{a}$ values.

\subsection{Application of assay to characterize membrane surface modifications}

The method was used to characterize two surface modifications performed on SW30XLE membrane samples. The first modification, EDC/NHS-mediated coupling of ethylamine with residual carboxyl groups, sought to decrease the carboxyl density. EDC/NHS-mediated coupling is commonly used to graft functional materials to the surface of TFC membranes [35]. The second modification, grafting polyacrylic acid to the surface, sought to increase the carboxyl density.

\subsubsection{EDC/NHS-mediated coupling of ethylamine}

Coupling of ethylamine decreased the total carboxyl density from 9.3 sites $/ \mathrm{nm}^{2}$ to 6.9 sites $/ \mathrm{nm}^{2}$, with the carboxyl density associated with $\mathrm{p} K_{a, 1}$ decreasing to near-zero, while the carboxyl density associated with $\mathrm{p} K_{a, 2}$ was unaffected (Fig. 7). As discussed in Section 3.2, one possible interpretation of the ionization behavior of polyamide films is that $\mathrm{p} K_{a, 1}$ is associated with carboxyl groups on the membrane surface, while $\mathrm{p} K_{a, 2}$ is associated with carboxyl groups buried within the polyamide polymer network. The change in ionization 
behavior for the ethylamine-modified membrane supports this interpretation; EDC/NHS-mediated reactions are unlikely to occur within the polyamide film due to the bulky size of EDC (155 g/mol), which is further enlarged due to its positive charge at the $\mathrm{pH}$ of the coupling reaction. Ionized EDC would likely be sterically excluded from entering the polyamide polymer network, restricting reactions to carboxyl groups on the membrane surface. Thus, the results for the ethylamine-modified membrane suggest that surface-exposed carboxyl groups are associated with $\mathrm{p} K_{a, 1}$, while buried carboxyl groups that are inaccessible to EDC are associated with $\mathrm{p} K_{a, 2}$.

\subsubsection{Grafting of polyacrylic acid}

Redox-initiated polymerization of acrylic acid was used to graft polyacrylic acid to the TFC membrane. Successful grafting of polyacrylic acid was qualitatively demonstrated by infrared spectroscopy (Fig. S2). The absolute carboxyl density, and thus the monomer density of the grafted polymer, was then determined using the developed method. The carboxyl density increased sharply from 9.3 sites $/ \mathrm{nm}^{2}$ to 37.2 sites $/ \mathrm{nm}^{2}$, indicating a grafted polymer concentration of 26.8 acrylic acid units $/ \mathrm{nm}^{2}$ (Fig. 7). Densities associated with $\mathrm{p} K_{a, 1}$ and $\mathrm{p} K_{a, 2}$ both increased, with increases of 8.4 and 18.2 sites $/ \mathrm{nm}^{2}$, respectively. If the physical interpretation in Sections 3.2 and 3.4 .1 of the ionization behavior is accurate, then the increased densities indicate that polyacrylic acid grew on the surface and within the polyamide film. In a microscopy study using the same initiators and monomer, polyacrylic acid was found to grow within the support layer of a commercial NF membrane, suggesting that the reactants can penetrate through the polyamide layer of NF membranes [47]. Similar penetration is likely for the RO membrane tested here, which would enable polymer growth in small voids within the polyamide film. Such growth would explain the increased density associated with $\mathrm{p} K_{a, 2}$.

More importantly, the result demonstrates the ability of the method to not only show successful grafting, but also quantify the absolute grafting density of negatively-charged functional materials (e.g., polymer brush layers) on membranes and other surfaces. In comparison, infrared spectroscopy typically only yields relative densities [47]. Characterization of grafted materials with weak-acid functional groups (e.g., carboxyl and 
phenolic moieties) can directly follow the bind-and-elute method described here. Characterization of grafted materials with strong-acid functional groups (e.g., sulfonate moieties) that are not expected to re-protonate in $1 \%$ nitric acid may need to use microwave digestion or an alternative elution method.

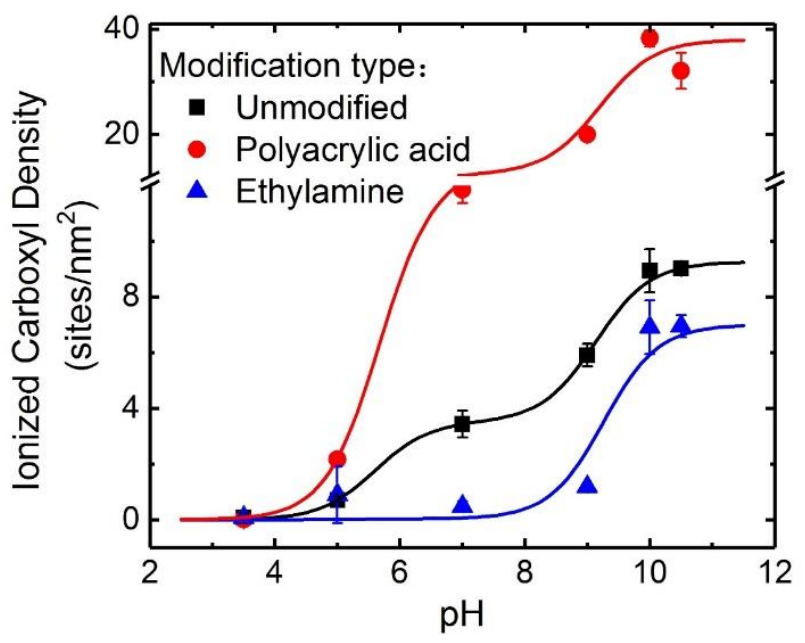

Fig. 7. Ionized carboxyl density at varying $\mathrm{pH}$ for an unmodified membrane (SW30XLE, Dow), a membrane for which residual carboxyl groups were reacted with ethylamine, and a membrane with grafted polyacrylic acid. Surface modifications followed the procedures in Section 2.7. Curves were fit using Eq. 3. The polyester fabric was removed for all samples.

\section{Conclusion}

A simple, accessible and reproducible assay was developed and verified to allow accurate quantitation of the carboxyl group density of the polyamide layer of TFC membranes. The developed assay combines the accuracy and precision of RBS-based techniques with simple bind-and-elute sample preparation and ICP-MS analysis. RBS-based methods and the method developed here both use silver as an ion probe and allow quantitative characterization of the fixed charge density and ionization behavior. The main advantage of the method described in this study is the widespread accessibility of ICP-MS, which contrasts sharply with the inaccessibility of RBS instruments. We believe that the method will find extensive usage, including but not limited to studies on the effects of membrane surface charge and/or surface modifications (e.g., charged polymer brush layers) 
on separation performance, fouling, and scaling. Importantly, it could also be readily applied to assess the functional group density of other films and solid surfaces, which similarly cannot be determined by conventional titration methods.

In addition, an alternative explanation was proposed to describe the ionization behavior of polyamide films. In this interpretation, carboxyl groups that ionize at near neutral $\mathrm{pH}$ correspond to groups on the membrane surface, while carboxyl groups that ionize at $\mathrm{pH}$ above $\sim 8$ correspond to groups buried within the polyamide polymer network, regardless of the local pore size. While this interpretation is supported by the results for surface-modified membranes, further studies will be required to conclusively determine if the ionization behavior stems from this surface/buried mechanism, the previously proposed aggregate pore/network pore mechanism, or some completely different mechanism.

\section{Acknowledgments}

We acknowledge the support received from the National Science Foundation through the Engineering Research Center for Nanotechnology-Enabled Water Treatment (ERC-1449500) and via Grant CBET 1437630. We also acknowledge the National Science Foundation Graduate Research Fellowship awarded to J.R.W.

\section{References}

1. K.P. Lee, T.C. Arnot, D. Mattia, A review of reverse osmosis membrane materials for desalination-Development to date and future potential, J. Membr. Sci., 370 (2011) 1-22.

2. J.R. Werber, C.O. Osuji, M. Elimelech, Materials for next-generation desalination and water purification membranes, Nat. Rev. Mater., 1 (2016).

3. O. Coronell, B.J. Marinas, X.J. Zhang, D.G. Cahill, Quantification of functional groups and modeling of their ionization behavior in the active layer of FT30 reverse osmosis membrane, Environ. Sci. Technol., 42 (2008) 5260-5266.

4. A. Roy, T.L. Arrowood, A.S. Badami, R.C. Cieslinski, D.D. Hawn, S.D. Jons, M. Paul, S. Rosenberg, $\mathrm{H}$. Wu, Composite polyamide membrane with improved structure, U.S. Pat. No. 0165387, 2015.

5. V.T. Do, C.Y. Tang, M. Reinhard, J.O. Leckie, Degradation of polyamide nanofiltration and reverse osmosis membranes by hypochlorite, Environ. Sci. Technol., 46 (2012) 852-859.

6. S.D. Jons, K.J. Stutts, M.S. Ferritto, W.E. Mickols, Treatment of composite polyamide membranes to improve performance, U.S. Pat. No. 5876602, 1999. 
7. S. Bhattacharjee, J.C. Chen, M. Elimelech, Coupled model of concentration polarization and pore transport in crossflow nanofiltration, AIChE J., 47 (2001) 2733-2745.

8. X.L. Wang, T. Tsuru, S. Nakao, S. Kimura, The electrostatic and steric-hindrance model for the transport of charged solutes through nanofiltration membranes, J. Membr. Sci., 135 (1997) 19-32.

9. J. Schaep, B. Van der Bruggen, C. Vandecasteele, D. Wilms, Influence of ion size and charge in nanofiltration, Sep. Purif. Technol., 14 (1998) 155-162.

10. A.E. Yaroshchuk, Non-steric mechanisms of nanofiltration: superposition of Donnan and dielectric exclusion, Sep. Purif. Technol., 22-3 (2001) 143-158.

11. J. Schaep, C. Vandecasteele, Evaluating the charge of nanofiltration membranes, J. Membr. Sci., 188 (2001) 129-136.

12. J.T. Arena, M. Chwatko, H.A. Robillard, J.R. McCutcheon, pH Sensitivity of Ion Exchange through a Thin Film Composite Membrane in Forward Osmosis, Environ. Sci. Technol. Lett., 2 (2015) 177-182.

13. X. Lu, C. Boo, J. Ma, M. Elimelech, Bidirectional diffusion of ammonium and sodium cations in forward osmosis: role of membrane active layer surface chemistry and charge, Environ. Sci. Technol., 48 (2014) 14369-14376.

14. B. Mi, M. Elimelech, Gypsum scaling and cleaning in forward osmosis: measurements and mechanisms, Environ. Sci. Technol., 44 (2010) 2022-2028.

15. J. Wang, L. Wang, R. Miao, Y. Lv, X. Wang, X. Meng, R. Yang, X. Zhang, Enhanced gypsum scaling by organic fouling layer on nanofiltration membrane: Characteristics and mechanisms, Water Res., 91 (2016) 203-213.

16. S.K. Hong, M. Elimelech, Chemical and physical aspects of natural organic matter (NOM) fouling of nanofiltration membranes, J. Membr. Sci., 132 (1997) 159-181.

17. Y. Mo, A. Tiraferri, N.Y. Yip, A. Adout, X. Huang, M. Elimelech, Improved antifouling properties of polyamide nanofiltration membranes by reducing the density of surface carboxyl groups, Environ. Sci. Technol., 46 (2012) 13253-13261.

18. S.S. Deshmukh, A.E. Childress, Zeta potential of commercial RO membranes: influence of source water type and chemistry, Desalination, 140 (2001) 87-95.

19. M. Elimelech, W.H. Chen, J.J. Waypa, Measuring the Zeta (Electrokinetic) Potential of Reverse-Osmosis Membranes by a Streaming Potential Analyzer, Desalination, 95 (1994) 269-286.

20. S.L. Walker, S. Bhattacharjee, E.M.V. Hoek, M. Elimelech, A novel asymmetric clamping cell for measuring streaming potential of flat surfaces, Langmuir, 18 (2002) 2193-2198.

21. A. Tiraferri, M. Elimelech, Direct quantification of negatively charged functional groups on membrane surfaces, J. Membr. Sci., 389 (2012) 499-508.

22. M. Conato, F. Sumera, Biodegradable Polyesters and Polyamides From Difunctionalized Lauric and Coconut Fatty Acids, J. Polym. Environ., 20 (2012) 217-223.

23. O. Coronell, M.I. Gonzalez, B.J. Marinas, D.G. Cahill, Ionization behavior, stoichiometry of association, and accessibility of functional groups in the active layers of reverse osmosis and nanofiltration membranes, Environ. Sci. Technol., 44 (2010) 6808-6814.

24. O. Coronell, B.I. Marinas, D.G. Cahill, Accessibility and ion exchange stoichiometry of ionized carboxylic groups in the active layer of FT30 reverse osmosis membrane, Environ. Sci. Technol., 43 (2009) 5042-5048. 
25. O. Coronell, B.J. Marinas, D.G. Cahill, Depth heterogeneity of fully aromatic polyamide active layers in reverse osmosis and nanofiltration membranes, Environ. Sci. Technol., 45 (2011) 4513-4520.

26. C.C. Wamser, M.I. Gilbert, Detection of Surface Functional-Group Asymmetry in Interfacially-Polymerized Films by Contact-Angle Titrations, Langmuir, 8 (1992) 1608-1614.

27. H.X. He, W. Huang, H. Zhang, Q.G. Li, S.F.Y. Li, Z.F. Liu, Demonstration of high-resolution capability of chemical force titration via study of acid/base properties of a patterned self-assembled monolayer, Langmuir, 16 (2000) 517-521.

28. D.V. Vezenov, A. Noy, L.F. Rozsnyai, C.M. Lieber, Force titrations and ionization state sensitive imaging of functional groups in aqueous solutions by chemical force microscopy, J. Am. Chem. Soc., 119 (1997) 2006-2015.

29. L.A. Perry, O. Coronell, Reliable, bench-top measurements of charge density in the active layers of thin-film composite and nanocomposite membranes using quartz crystal microbalance technology, J. Membr. Sci., 429 (2013) 23-33.

30. S. Karan, Z. Jiang, A.G. Livingston, Sub-10 nm polyamide nanofilms with ultrafast solvent transport for molecular separation, Science, 348 (2015) 1347-1351.

31. C.Y.Y. Tang, Y.N. Kwon, J.O. Leckie, Probing the nano- and micro-scales of reverse osmosis membranes - A comprehensive characterization of physiochemical properties of uncoated and coated membranes by XPS, TEM, ATR-FTIR, and streaming potential measurements, J. Membr. Sci., 287 (2007) 146-156.

32. V. Freger, Swelling and morphology of the skin layer of polyamide composite membranes: an atomic force microscopy study, Environ. Sci. Technol., 38 (2004) 3168-3175.

33. S.L. Goertzen, K.D. Theriault, A.M. Oickle, A.C. Tarasuk, H.A. Andreas, Standardization of the Boehm titration. Part I. CO2 expulsion and endpoint determination, Carbon, 48 (2010) 1252-1261.

34. A. Vaisanen, R. Suontamo, J. Silvonen, J. Rintala, Ultrasound-assisted extraction in the determination of arsenic, cadmium, copper, lead, and silver in contaminated soil samples by inductively coupled plasma atomic emission spectrometry, Anal. Bioanal. Chem., 373 (2002) 93-97.

35. A. Tiraferri, C.D. Vecitis, M. Elimelech, Covalent binding of single-walled carbon nanotubes to polyamide membranes for antimicrobial surface properties, ACS Appl. Mater. Interfaces, 3 (2011) 2869-2877.

36. S. Belfer, Y. Purinson, O. Kedem, Surface modification of commercial polyamide reverse osmosis membranes by radical grafting: An ATR- FTIR study, Acta Polym., 49 (1998) 574-582.

37. S.H. Kim, S.Y. Kwak, T. Suzuki, Positron annihilation spectroscopic evidence to demonstrate the flux-enhancement mechanism in morphology-controlled thin-film-composite (TFC) membrane, Environ. Sci. Technol., 39 (2005) 1764-1770.

38. T. Fujioka, N. Oshima, R. Suzuki, W.E. Price, L.D. Nghiem, Probing the internal structure of reverse osmosis membranes by positron annihilation spectroscopy: Gaining more insight into the transport of water and small solutes, J. Membr. Sci., 486 (2015) 106-118.

39. M. Shen, S. Keten, R.M. Lueptow, Dynamics of water and solute transport in polymeric 
reverse osmosis membranes via molecular dynamics simulations, J. Membr. Sci., 506 (2016) 95-108.

40. K.J. Lamble, S.J. Hill, Microwave digestion procedures for environmental matrices, Analyst, 123 (1998) 103R-133R.

41. L. Lin, R. Lopez, G.Z. Ramon, O. Coronell, Investigating the void structure of the polyamide active layers of thin-film composite membranes, J. Membr. Sci., 497 (2016) 365-376.

42. M.M. Klosowski, C.M. McGilvery, Y.Q. Li, P. Abellan, Q. Ramasse, J.T. Cabral, A.G. Livingston, A.E. Porter, Micro-to nano-scale characterisation of polyamide structures of the SW30HR RO membrane using advanced electron microscopy and stain tracers, J. Membr. Sci., 520 (2016) 465-476.

43. F.G. Helfferich, Ion Exchange, McGraw-Hill, New York, NY, 1962.

44. S. Bason, Y. Oren, V. Freger, Ion transport in the polyamide layer of RO membranes: Composite membranes and free-standing films, J. Membr. Sci., 367 (2011) 119-126.

45. J. Muscatello, E.A. Müller, A.A. Mostofi, A.P. Sutton, Multiscale molecular simulations of the formation and structure of polyamide membranes created by interfacial polymerization, J. Membr. Sci., 527 (2016) 180-190.

46. M.F. Jimenez Solomon, Y. Bhole, A.G. Livingston, High flux membranes for organic solvent nanofiltration (OSN)-Interfacial polymerization with solvent activation, J. Membr. Sci., 423 (2012) 371-382.

47. V. Freger, J. Gilron, S. Belfer, TFC polyamide membranes modified by grafting of hydrophilic polymers: an FT-IR/AFM/TEM study, J. Membr. Sci., 209 (2002) 283-292. 